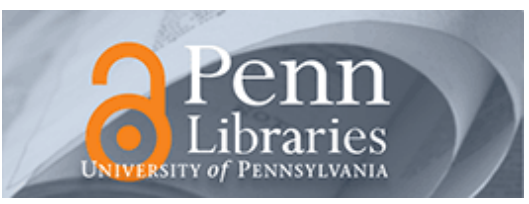

University of Pennsylvania

ScholarlyCommons

September 2004

\title{
Toward a 6 DOF Body State Estimator for a Hexapod Robot with Dynamical Gaits
}

\author{
Pei-Chun Lin \\ University of Michigan \\ Haldun Komsuoglu \\ University of Michigan \\ Daniel E. Koditschek \\ University of Pennsylvania, kod@seas.upenn.edu
}

Follow this and additional works at: https://repository.upenn.edu/ese_papers

\section{Recommended Citation \\ Pei-Chun Lin, Haldun Komsuoglu, and Daniel E. Koditschek, "Toward a 6 DOF Body State Estimator for a Hexapod Robot with Dynamical Gaits", . September 2004.}

Copyright 2004 IEEE. Reprinted from Proceedings of the 2004 IEEE/RSJ International Conference on Intelligent Robots and Systems (IROS 2004), Volume 3, pages 2265-2270.

This material is posted here with permission of the IEEE. Such permission of the IEEE does not in any way imply IEEE endorsement of any of the University of Pennsylvania's products or services. Internal or personal use of this material is permitted. However, permission to reprint/republish this material for advertising or promotional purposes or for creating new collective works for resale or redistribution must be obtained from the IEEE by writing to pubs-permissions@ieee.org. By choosing to view this document, you agree to all provisions of the copyright laws protecting it.

NOTE: At the time of publication, author Daniel Koditschek was affiliated with the University of Michigan. Currently (August 2005), he is a faculty member in the Department of Electrical and Systems Engineering at the University of Pennsylvania.

This paper is posted at ScholarlyCommons. https://repository.upenn.edu/ese_papers/132

For more information, please contact repository@pobox.upenn.edu. 


\title{
Toward a 6 DOF Body State Estimator for a Hexapod Robot with Dynamical Gaits
}

\begin{abstract}
We report on a continuous time full body state estimator for a hexapod robot operating in the dynamical regime (entailing a significant aerial phase) on level ground that combines a conventional rate gyro with a novel leg strain based body pose estimator. We implement this estimation procedure on the robot RHex and evaluate its performance using a visual ground truth measurement system. As an independent assessment of our estimator's quality we also compare its odometry performance to sensorless averaged open loop distance-per-stride estimates.

\section{Comments}

Copyright 2004 IEEE. Reprinted from Proceedings of the 2004 IEEE/RSJ International Conference on Intelligent Robots and Systems (IROS 2004), Volume 3, pages 2265-2270.

This material is posted here with permission of the IEEE. Such permission of the IEEE does not in any way imply IEEE endorsement of any of the University of Pennsylvania's products or services. Internal or personal use of this material is permitted. However, permission to reprint/republish this material for advertising or promotional purposes or for creating new collective works for resale or redistribution must be obtained from the IEEE by writing to pubs-permissions@ieee.org. By choosing to view this document, you agree to all provisions of the copyright laws protecting it.

NOTE: At the time of publication, author Daniel Koditschek was affiliated with the University of Michigan. Currently (August 2005), he is a faculty member in the Department of Electrical and Systems Engineering at the University of Pennsylvania.
\end{abstract}




\section{Toward a 6 DOF Body State Estimator for a Hexapod Robot with Dynamical Gaits}

\author{
Pei-Chun Lin \\ Department of Mechanical Engineering \\ The University of Michigan \\ Ann Arbor, Michigan, USA \\ Email: pclin@umich.edu
}

\author{
Haldun Komsuoğlu, Daniel E. Koditschek \\ Department of Electrical Engineering and Computer Science \\ The University of Michigan \\ Ann Arbor, Michigan, USA \\ Email: \{hkomsuog, kod\}@umich.edu
}

\begin{abstract}
We report on a continuous time full body state estimator for a hexapod robot operating in the dynamical regime (entailing a significant aerial phase) on level ground that combines a conventional rate gyro with a novel leg strain based body pose estimator. We implement this estimation procedure on the robot RHex and evaluate its performance using a visual ground truth measurement system. As an independent assessment of our estimator's quality we also compare its odometry performance to sensorless averaged open loop distance-per-stride estimates.
\end{abstract}

\section{INTRODUCTION}

The hexapod, RHex [1], exhibits unprecedented mobility for a legged autonomous robot [2]. Using an open loop feedforward control strategy, the machine runs at speeds exceeding five body lengths per second on even terrain [3], and negotiates badly broken and unstable surfaces, as well as stairs [4]-[6].

In our initial studies with sensor based controllers we have observed significant behavioral improvement from even minimal feedback [7], [8]. Theoretical and simulation evidence [9] suggests that the availability of full body state estimates as well as force interactions with the surrounding environment throughout the stance and aerial phases of locomotion, should confer considerably greater agility still.

Building a sensor suite that can deliver full body state information - six configuration coordinates together with their six time derivatives - at data rates relevant to motor control $(\sim 1 \mathrm{kHz})$ remains a challenging problem in legged robotics because of the constraints upon onboard instrumentation combined with extreme variations in operating regime. The traditional inertial measurement unit (IMU) for rigid bodies in flight typically lies out of the range of robotics applications because of its cost and excessive volume. Appropriately cheap and small IMU packages typically suffer severe drift and saturation. Moreover, while ballistic flight models are quite accurate, legged machines by definition spend a large fraction of their locomotion duty cycle in ground contact. There, the determination of an appropriate model is greatly complicated by the uncertainty in ground conditions (local terrain shape, slipperiness, and damping and compliance properties) and leg contact conditions (which legs are in stance).

Recently, we have introduced a novel leg-strain based full body pose estimator (hereafter referred to as the "leg pose sensor") for the tripod stance phase of a hexapedal robot [10]. In that work we have demonstrated that a memoryless transformation built from (data driven phenomenological) models relating leg strain to configuration coupled with a conventional kinematic model of leg configuration to body pose can yield the six coordinates of body position and orientation when the robot's three legs are fixed in the ground. In this paper, we join to the leg pose sensor a 3 degree of freedom (DOF) rate gyro to develop a very simple full body state estimator that operates continuously during flight, and touchdown and liftoff transients as well as during the full tripod stance phase of steady state hexapod dynamical running ${ }^{1}$. By this latter term we denote stable periodic legged locomotion with a significant aerial phase - $25 \%$ of the complete stride for the "jogging" gait adopted in the accompanying empirical study ${ }^{2}$. This represents an important first step in a general full body state estimator we are presently developing by combining accelerometers as well.

In walking gaits with no aerial phase, complete 6 DOF body pose in continuous time can be derived in principle from a purely kinematic model [12] without velocity state estimation. In contrast, a robot operating in a jogging gait with significant aerial phase would seem to require full state estimation - both velocity and configuration information. In order to build such estimators, of course, the sensor suite must incorporate enough information to allow the reconstruction of full state from the record of past measurements filled in by some dynamical model. In the present paper we take a partial step toward this goal by combing gyro data with our leg strain data through a naive kinematic model. Roughly speaking, the gyro is used to augment the leg sensor data during flight and the leg sensor is used to recalibrate the gyro that suffers the well known problems of saturation and drift in stance. Explicit dynamical modeling promises to be complicated since the physical robot acts as a lagrangian system with $3^{6}$ different models depending on touchdown-stick/touchdownslip/liftoff conditions on each leg. Instead, we simplify that problem by using three repeatedly successive models - tripod stance phase ${ }^{3}$, aerial phase, and transient phase ${ }^{4}$ as a starting point to describe this jogging locomotion and to estimate full body state by models with partial true state obtained from leg sensor and gyro.

The idea of sensor fusion bas widely spread into many different fields, and mobile robotics, typically wheel vehicles, is one of the stereo type - from algorithm [13] or

\footnotetext{
${ }^{1}$ In this preliminary study, to ease the computational load on the onboard processor, all gyro data has been integrated offine and combined onboard processor, all gyro data has been in
with the leg pose sensor on the benchiop.

${ }^{2}$ Note that hexapedal running gaits need not entail an aerial phase to be "dynamical" in the sense of rcquiring careful management of kinetic energy 10 insure balance and stcady progress [11]. However, for purposes of our sensor suite, gaits with no aerial phase would in principlc be completcly covered by the leg strain based subsystem.

${ }^{3}$ Denotes the mode of leg contact wherein the three toes of the front and rear ipsilateral legs and the middle contralateral leg of a tripod"are all in contact with the ground.

${ }^{4}$ Denotes the conditions besides tripod stance phase and aerial phase.
} 


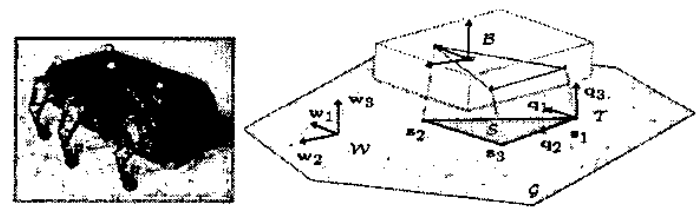

Fig. 1. (Left) RHex with strain based leg pose sensor. (Right) Sketch illustrating the robot on a fal ground plane. $\mathcal{G}$, within a tripod stance phase during which it is supported by only three legs whose toes defi ne the support triangle, $\mathcal{S}$. Attached to the support triangle, we defi ne a tripod coordinate system, $\mathcal{T}$. Figure is taken from [10].

controller design [14] to practical implementation, like fusion inertia and vision information [15], [16] or fusion gyro and other sensors [17]. With support from multi sensors reliability and performance of robot improves significantly, like in positioning estimation or error reduction [18]. For legged robots, sensor fusion is addressed in many aspects as well, from typical positioning (by sonar sensors in [19]), to inertial or vision guidance [20], even to entertainment robots [21], [22]. However, from the database ${ }^{5}$ we haven't seen any paper related to full body state for a hexapod with dynamical gaits with data from gyro and leg pose sensor, which indicates the novelty of our work.

Section II introduces the algorithm for body pose in a local coordinate frame from the leg pose sensor during tripod stance phase. This material originally introduced in [10] is briefly reviewed here for ease of exposition. Section III presents the algorithm for continuous time 6 DOF body state estimation using sensor inputs from the leg pose sensor and gyro. Section IV examines the accuracy of the resulting body state estimator implemented on RHex pictured in Figure 1(right) equipped with our previously developed leg pose sensor [10] and commercial 3 DOF rate gyro (Fizoptika VG941-3A). The performance is assessed with respect to an independent visual ground truth measurement system (GTMS) initially introduced in [10] and detailed in [12].

\section{BODY POSE FROM LEG STRAIN DURING TRIPOD STANCE Phase}

A typical "tripod stance" during robot locomotion is depicted in Figure 1(right). It is intuitively clear that knowledge of the configuration relative to the body of each leg in contact with the ground, together with information about the ground contact points yields complete pose information. By defining the tripod coordinate system, $\mathcal{T}$, detailed in [10], we can construct a rigid transformation, $\mathbf{H}_{T B}: \mathcal{B} \rightarrow \mathcal{T}$, relating the body coordinate system, $\mathcal{B}$, to the tripod coordinate system, $\mathcal{T}$,

$$
\mathbf{H}_{T B}:=\left[\begin{array}{cc}
\mathbf{R}_{l} & \mathbf{s}_{1} \\
0 & 1
\end{array}\right] .
$$

where $s_{1}$ denotes center of mass (COM) translation and $\mathbf{R}_{1}$ denotes a rotation matrix, which can also be represented in three Euler angles - pitch $\left(\alpha_{l}\right)$, roll $\left(\beta_{l}\right)$, and yaw $\left(\gamma_{l}\right)$.

During a tripod stance phase this algorithm continuously delivers 6 DOF body pose in tripod coordinates. The tripod coordinate system, $\mathcal{T}$, has a fixed rigid relationship to world coordinates assuming no toe slippage, permitting for example, complete odometry during steady walking

\footnotetext{
${ }^{5}$ We have searched the Compendex and IEEExplore data bases using the key words 'Legged Robot", 'Sensor Fusion".
}

gaits (i.e., alternation of leg contact configuration between single and double tripod stance) as we have presented in [23]. When slipping occurs, additional sensors (e.g., the traditional IMU suite discussed above) are required to measure the time varying transformation between tripod coordinate system, $\mathcal{T}$, and the world coordinates.

Because of its relatively constrained kinematics (only one actuator for each of its six compliant legs), implementation of this scheme on RHex cannot capture changes in yaw. This limitation notwithstanding, we have shown that the leg strain sensor suite successfully delivers 5 DOF body pose data on RHex, not only in single tripod stance but also in continuous walking at various speeds [23]. However the absence of any yaw data, combined with the deleterious effects of toe slippage and the importance of aerial phase gaits in RHex's locomotion behavioral suite all lend strength to the motivation for adding more sensors. For example, even in steady walking gaits, this leg strain based body pose sensor cannot compute complete odometry since the only way to tum RHex's body is to initiate toe slipping.

\section{Computation of Continuous Time Body STATE}

We now introduce a naive algorithm for combining the leg pose sensor (providing intermittent continuous time information about body configuration during stance) with rate gyro based body attitude measurements to yield body state with respect to an inertial frame located at the initiation of the robot's motion that we take to be the world coordinates which we express using the homogeneous matrix representation as

$$
\mathbf{H}_{W B}:=\left[\begin{array}{cc}
\mathbf{R} & \mathbf{r} \\
0 & 1
\end{array}\right]
$$

where $\mathbf{r}:=\left[r_{x} r_{y} r_{z}\right]^{T}$ denotes COM translation with three components in lateral $\left(r_{x}\right)$, fore/aft $\left(r_{y}\right)$, and vertical $\left(r_{z}\right)$ directions and $\mathbf{R}$ denotes a rotation matrix, related by three Euler angles pitch $(\alpha)$, roll $(\beta)$, and yaw $(\gamma)$ by a function [24], $\mathcal{R}$, with definition $\mathbf{R}:=\mathcal{R}(\alpha, \beta, \gamma)$.

Consider the typical sequence of leg contact conditions, depicted in Figure 2, that occur during steady state operation in a stable running gait. During the $i^{\text {th }}$ stride interval, $C(i):=\left[t_{1}(i) t_{5}(i)\right] \subset R$, a tripod stance interval, $\Phi_{S}(i):=\left[t_{1}(i) t_{2}(i)\right]$, is succeeded by a period of time when the legs begin to liftoff, $\Phi_{L}(i):=\left[t_{2}(i) t_{3}(i)\right]$, followed by an interval of aerial fight, $\Phi_{A}(i):=\left[t_{3}(i) t_{4}(i)\right]$, then touching down through another period of varied leg contacts, $\Phi_{T}(i):=\left[t_{4}(i) t_{5}(i)\right]$, to the fixed tripod stance interval $\Phi_{S}(i+1)$ of the next stride, $C(i+1)$. We conceive of the liftoff and touchdown intervals, $\Phi_{L}(i), \Phi_{T}(i)$ as "transients" because they typically exhibit complex sequences of successive leg contacts that reveal little consistent pattern from run to run (or, often, even from stride to stride). Our algorithm for combining rate gyro with leg pose sensor data assumes perfect information about which phase interval the robot is undergoing at every instant of time. In our implementation, the crucial leg contact information required to detect the onset and termination of each of these phases of a stride may be gleaned directly from the individual leg strain sensors.

We further assume the existence of a continuously available gyro integrator output signal that delivers the 3 DOF body attitude data throughout the entirety of the each stride at the same rate as the body pose sensor. In this work we follow Skaff et al [20] and implement an 


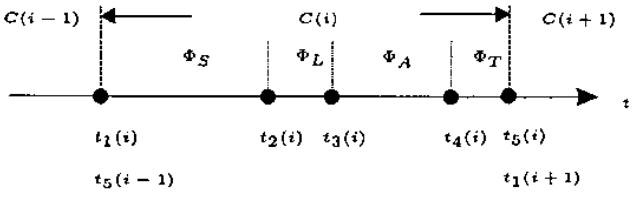

Fig. 2. Four consecutive intervals during the $i^{\text {th }}$ stride, $C(i)$.

integrator operating at the same $300 \mathrm{~Hz}$ rate as our leg pose sensor system. For purposes of presentation, we will find it convenient sometimes to refer to the integrator output signal in the form of a rotation matrix, $\mathbf{R}_{g}(t)$, relating the body orientation to an inertial frame, and sometimes to use the three Euler angles - pitch, $\alpha_{g}(t)$, roll, $\beta_{g}(t)$, and yaw $\gamma_{g}(t)$.

Generally, one expects that accumulated integration error will gradually degrade the body orientation signal delivered by the gyro system. In contrast, while it is only active during the tripod stance interval, $\Phi_{S}(i)$, the leg pose system performs a memoryless kinematic transformation of its data, including all translational and rotational (except yaw) components (1) with a fixed accuracy bound, hence no possibility for drift. Moreover, assuming level ground as we do, three of these stance pose components - pitch $(\alpha)$, roll $(\beta)$, and height $\left(r_{z}\right)$ - coincide with the inertial frame. The other components - lateral $\left(r_{x}\right)$ and fore/aft $\left(r_{y}\right)$ translation and yaw $(\gamma)-$ are relative to the present stance frame only. Combining the favorable characteristics of the two different sensors, we treat $r_{z}, \alpha$, and $\beta$ as "memoryless" state which is reset during every tripod stance interval, $\Phi_{S}(i)$, and while (unavoidably) treating $r_{x}, r_{y}$, and $\gamma$ as state relating to whole history because of absence of true positioning sensor like camera or GPS. Without data from other sensor source available, yaw, $\gamma(t)$, is directly inherited from $\gamma_{g}(t)$ (i.e. $\gamma(t)=\gamma_{g}(t)$ ) from gyro for all intervals.

With these assumptions and notation in place, the algorithm can now be presented in sequence of intervals as follows.

\section{A. Body State during Tripod Stance Interval, $\Phi_{S}$}

Without any loss of generality, in the $i^{\text {th }}$ stride, $C(i)$, we can assume the homogeneous transformation $\mathbf{H}_{W B}\left(t_{1}(i)\right)$ at touchdown moment $t_{1}(i)$ as known state obtained from previous $(i-1)^{t h}$ stride, $C(i-1)$. With the memoryless state $r_{z}, \alpha$, and $\beta$ available from leg sensor during this interval, a "reset" initial homogeneous transformation at $t_{1}(i)$, the beginning of the tripod stance interval, can be defined as

$$
\mathbf{H}_{0}^{S}(i):=\left[\begin{array}{cc}
I & \mathbf{r}_{0} \\
0 & 1
\end{array}\right]
$$

where $\mathbf{r}_{0}:=\left[r_{x}\left(t_{1}(i)\right) r_{y}\left(t_{1}(i)\right) 0\right]^{T}$ preserves the history of planar translation which can be extracted from $\mathbf{H}_{W B}\left(t_{1}(i)\right)$ according to the notation defined in (2). This homogeneous transformation, $\mathbf{H}_{0}^{S}(i)$, keeps a link for state required history information but gets rid of the memoryless state which can be obtained during this tripod stance interval ${ }^{6}$.

${ }^{6}$ No requirement to preserve yaw state in this initial matrix because it is automatically recovered in the following computation when $\gamma_{g}(t)$ from gyro is imported.
From $\mathbf{H}_{T B}(t)$ (1) combined with yaw from gyro, $\gamma_{g}(t)$, we can construct the homogeneous transformation relating the body coordinate system at any instant, $t$, to that at tripod touchdown moment, $t_{1}(i)$, in world coordinate sense by

$$
\begin{array}{r}
\mathbf{H}_{B B}(t):=\left[\begin{array}{cc}
\mathbf{R}_{\gamma}(t) & 0 \\
0 & 1
\end{array}\right] \mathbf{H}_{T B}\left(t_{1}(i)\right)^{-1} \mathbf{H}_{T B}(t) \\
t \in\left[t_{1}(i) t_{2}(i)\right]
\end{array}
$$

where $\mathbf{R}_{\gamma}(t):=\mathcal{R}\left(0,0,\left(\gamma_{l}(t)-\gamma_{g}(t)\right)\right)$ denotes the yaw correction required to maintain the rotation in the tripod coordinate system, $\mathcal{T}$. When the toes do not slip (i.e. yaw motion can also be correctly detected by leg pose sensor during tripod stance interval, $\mathbf{R}_{\gamma}=I$, the tripod coordinate system, $\mathcal{T}$, is a fixed inertial frame in the ground, and the COM translation between any instant $t$ and touchdown moment $t_{1}(i)$ derived from (3) can be transformed without assistance of yaw data from other sensor suite to world coordinates by a simple geometry relation as we have explained in the leg pose sensor discussion of the previous section. In general, with toe slippage, the transformation back to world coordinates requires a complementary sensor suite to provide information regarding yaw rotation and rotation center at every instant. While yaw can be provided by the gyro, $\gamma_{g}(t)$, it is difficult to detect the rotation center without any toe force sensor. Thus we simply assume this rotation motion with respect to COM yields the computation shown in (3).

The COM translation in lateral $\left(r_{x}^{\mathbf{H}_{B B}}(t)\right)$ and in fore/aft $\left(r_{y}^{\mathbf{H}_{B B}}(t)\right)$ directions with respect to COM location at initial tripod touchdown moment, $t_{1}(i)$, can be extracted from $\mathbf{H}_{B B}(t)$ shown in (3) by the notation defined in (2). The COM vertical translation $\left(r_{z}^{\mathbf{H}_{T B}}(t)\right)$ is directly extracted from $\mathbf{H}_{T B}(t)$ (1) because, as we have mentioned above, it preserves non-drifting absolute height to the level ground. The rotation matrix, $\mathbf{R}^{S}(t)$, is constructed as $\mathbf{R}^{S}(t)=\mathcal{R}\left(\alpha_{l}(t), \beta_{l}(t), \gamma_{g}(t)\right)$ combining the leg pose sensor's pitch and roll data with the gyro's yaw data as explained above. In summary, the homogeneous transformation, $\mathbf{H}_{W B}(t)$, relating the body coordinate system to world coordinates during tripod stance interval, $\Phi_{S}$, is expressed as

$$
\begin{aligned}
& \mathbf{H}_{W B}(t):=\mathbf{H}_{0}^{S}(i)\left[\begin{array}{cc}
\mathbf{R}^{S}(t) & \mathbf{r}^{S}(t) \\
0 & 1
\end{array}\right] \quad t \in\left[t_{1}(i) t_{2}(i)\right] \\
& \text { where } \mathbf{r}^{S}(t):=\left[r_{x}^{\mathbf{H}_{B B}}(t) r_{y}^{\mathbf{H}_{B B}}(t) r_{z}^{\mathbf{H}_{T B}}(t)\right]^{T} .
\end{aligned}
$$

\section{B. Body State during Liftoff Transient, $\Phi_{L}$}

During liftoff transient interval, $\Phi_{L}(i)$, translational motion is modeled as "constant speed" motion with initial velocity $\dot{\mathbf{r}}_{0}^{L}$ estimated by differentiating (suitably smoothed) leg pose translation signals during the final portion of the previous tripod stance interval, $\Phi_{S}(i)$. Integrating forward using this naive zero acceleration model yields the following COM translation prediction at any instant, $t$, with respect to the initial liftoff time, $t_{2}(i)$, as $\mathbf{r}^{L}(t)=$ $\dot{\mathbf{r}}_{0}^{L}\left(t_{2}(i)\right)\left(t-t_{2}(i)\right)$ where $t \in\left[t_{2}(i) t_{3}(i)\right]$.

In contrast, there is a direct gyro reading for orientation during the transient periods, and, after reinitializing the rate gyro integrator with the leg pose sensor's orientation data at end of stance, we simply adopt the gyro's 
integrated signal via the rotation matrix, $\mathbf{R}_{l g}{ }^{7}, \mathbf{R}_{l g}:=$ $\mathbf{R}_{l}\left(t_{2}(i)\right) \mathbf{R}_{g}\left(t_{2}(i)\right)^{-1}$ at liftoff time $t_{2}(i)$ which indicates the last instant with the availability of two rotation matrices from both sensors, $\mathbf{R}_{g}(t)$ and $\mathbf{R}_{l}(t)^{8}$. The "relation" pitch $\left(\alpha^{\mathbf{R}_{i g}}(t)\right)$ and roll $\left(\beta^{\mathbf{R}_{i g}}(t)\right)$ between $\mathbf{R}_{g}(t)$ and $\mathbf{R}_{l}(t)$ can be extracted from $\mathbf{R}_{l g}$ by inverting the kinematic relationship [24], which allows us to construct the correct "recalibration" rotation matrix, $\mathbf{R}_{0}^{L}$, without resetting yaw motion defined as $\mathbf{R}_{0}:=\mathcal{R}\left(\alpha^{\mathbf{R}_{l g}}(t), \beta^{\mathbf{R}_{l g}}(t), 0\right)$. Thus, with the "reset" initial homogeneous transformation matrix,

$$
\mathbf{H}_{0}^{L}(i):=\left[\begin{array}{cc}
\mathbf{R}_{0} & \mathbf{r}\left(t_{2}(i)\right) \\
0 & 1
\end{array}\right]
$$

where $\mathbf{r}\left(t_{2}(i)\right)$ is obtained according to the notation shown in (2), the homogeneous transformation, $\mathbf{H}_{W B}(t)$, relating the body coordinate system to world coordinates during liftoff transient interval, $\Phi_{L}$, can be expressed as

$$
\mathbf{H}_{W B}(t):=\mathbf{H}_{0}^{L}(i)\left[\begin{array}{cc}
\mathbf{R}_{g}(t) & \mathbf{r}^{L}(t) \\
0 & 1
\end{array}\right] \quad t \in\left[t_{2}(i) t_{3}(i)\right]
$$

\section{Body State during Aerial flight, $\Phi_{A}$}

Translation trajectories during aerial phase, $\Phi_{A}(i)$ are predicted using the standard ballistic flight model, resulting in the COM translation at any instant, $t$, with respect to the initial liftoff time, $t_{3}(i)$, as $\mathbf{r}^{A}(t)=\dot{\mathbf{r}}_{0}^{L}\left(t_{2}(i)\right)(t-$ $\left.t_{3}(i)\right)+(1 / 2) G_{(}\left(t-t_{3}(i)\right)^{2}$ where $t \in\left[t_{3}(i) t_{4}(i)\right]$ and $\mathbf{G}=[00-g]^{T}$ with gravity constant, $g$. Similar to the procedure in liftoff transients, with the newly defined "reset" initial homogeneous transformation matrix defined as,

$$
\mathbf{H}_{0}^{A}(i):=\left[\begin{array}{cc}
\mathbf{R}_{0} & \mathbf{r}\left(t_{3}(i)\right) \\
0 & 1
\end{array}\right]
$$

we can construct the homogeneous transformation, $\mathbf{H}_{W_{B}}(t)$, relating the body coordinate system to world coordinates during aerial flight, $\Phi_{A}$, as

$$
\mathbf{H}_{W B}(t):=\mathbf{H}_{0}^{A}(i)\left[\begin{array}{cc}
\mathbf{R}_{g}(t) & \mathbf{r}^{A}(t) \\
0 & 1
\end{array}\right] \quad t \in\left[t_{3}(i) t_{4}(i)\right]
$$

D. Body State during Touchdown transient, $\Phi_{T}$

With the same "constant speed" model, the computation procedure for body state during the touchdown transient interval, $\boldsymbol{\Phi}_{T}(i)$, is similar to that in liftoff transient interval, $\Phi_{L}(i)$. The only difference is the initial velocity in vertical direction due to the effect of gravity during aerial flight.

\section{EXPERIMENTAL RESULTS}

A. Performance Comparison of Pitch and Roll between Algorithm and Direct Gyro Integrator

To assess performance improvements resulting from the combination of leg pose with the rate gyro, we have run the robot RHex under the Ground Truth Measurement System (GTMS) - the independent visual ground truth measurement system introduced in [10] and detailed in

\footnotetext{
${ }^{7}$ This relation matrix, $\mathbf{R}_{l g}(t)$, is constant and effective during the whole "hon-tripod intervals" $\left(t \in\left[t_{2}(i) t_{5}(i)\right]\right)$ hecause of rigid hody locomotion.

${ }^{8}$ Comparing to the standard method which uses pitch and rolt at liftoff point as initial condition then to integratc, this "relation matrix" method is better in our situation because of the availability of $\mathbf{R}_{g}(t)$ with respect to initial point as well as saving the extra computation time.
}

TABLE I

NOISE FLOOR OF BODY POSE IN GTMS MEASUREMENTS

\begin{tabular}{|c||c|c|c|c|c|c|}
\hline State & $\begin{array}{c}r_{x} \\
(\mathrm{~cm})\end{array}$ & $\begin{array}{c}r_{y} \\
(\mathrm{~cm})\end{array}$ & $\begin{array}{c}r_{z} \\
(\mathrm{~cm})\end{array}$ & $\begin{array}{c}\alpha \\
(\mathrm{deg})\end{array}$ & $\begin{array}{c}\beta \\
(\mathrm{deg})\end{array}$ & $\begin{array}{c}\gamma \\
(\mathrm{deg})\end{array}$ \\
\hline Noise 66or & \pm 0.5 & \pm 0.5 & \pm 0.5 & \pm 1.71 & \pm 2.79 & \pm 1.30 \\
\hline
\end{tabular}

TABLE II

COMPARISON OF PITCH $(\alpha)$ AND ROLL $(\beta)$ IN RMS ERROR AND (RMS ERROR/GTMS NOISE FLOOR) RATIO

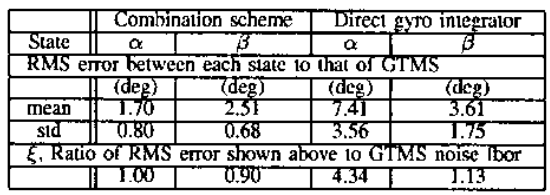

[12], whose noise floor statistics are listed relative to robot body state coordinates in Table I. This yields a comparison in pitch and roll between the raw gyro sensor and the combination leg-gyro sensor proposed in Section III. The steadily increasing drift of the raw gyro signal (plotted as a magenta dotted line) becomes visibly apparent over the $4 \mathrm{~m}$ run recorded in Figure 3 - particularly in pitch. Note, due to the limited size of the GTMS arena $(1.5 \mathrm{~m} \times 0.9 \mathrm{~m})$, the initial three quarters of this trial takes place outside its field of view, hence, the GTMS trace (green solid line) for pitch and roll only appears in this figure from roughly $t>7.5 \mathrm{~s}$. In contrast, the leg-gyro combination sensor (blue dashdotted line) maintains visibly better correspondence with the GTMS. We also quantify performance by presenting the standard root mean squared (RMS) error, given by $\chi(p, \hat{p}):=\sqrt{\left(\|p-\hat{p}\|_{2}^{2} / M\right)}$ where $p$ represents the state from GTMS; $\hat{p}$ denotes the same state from output of the algorithm; and $M$ is the length of the data. Table II lists the statistical results (mean and standard deviation from 10 experimental data) of RMS error between each state from both sources to that from GTMS, as well as $\xi$ - the ratio of this RMS error to the noise floor of GTMS . Smaller RMS Errors coordinated with validation checking from the ratio, $\xi$, indicate sensor combination scheme improves the performance. We suspect that the difference in magnitude of drift between the raw gyro pitch and roll data should be attributed to intrinsic sensor inconsistencies because we have confirmed they are working within a similar operating range with respect to angular rate, angular acceleration, and jerk.

\section{B. Performance of the Full Body State Estimator}

We now evaluate the performance of the body state estimation algorithm implemented on RHex [1], which is equipped with custom-designed leg pose sensor [10] and commercial 3 DOF rate gyro. Because the noise introduced differentiating the GTMS measurements is so severe, we are not able to check velocity estimates, and we limit our performance evaluation to assessing the quality of the onboard estimator's six position outputs relative to those recorded by the GTMS - the lateral $\left(r_{x}\right)$, fore-aft $\left(r_{y}\right)$, and vertical $\left(r_{z}\right)$ components of COM translation as well as pitch $(\alpha)$, roll $(\beta)$, and yaw $(\gamma)-$ all in world coordinates, $\mathcal{W}$. Figure 4 plots the comparison for each component over a typical run, which means the robot operation from standstill on one end of GTMS arena, jogging cross it to 

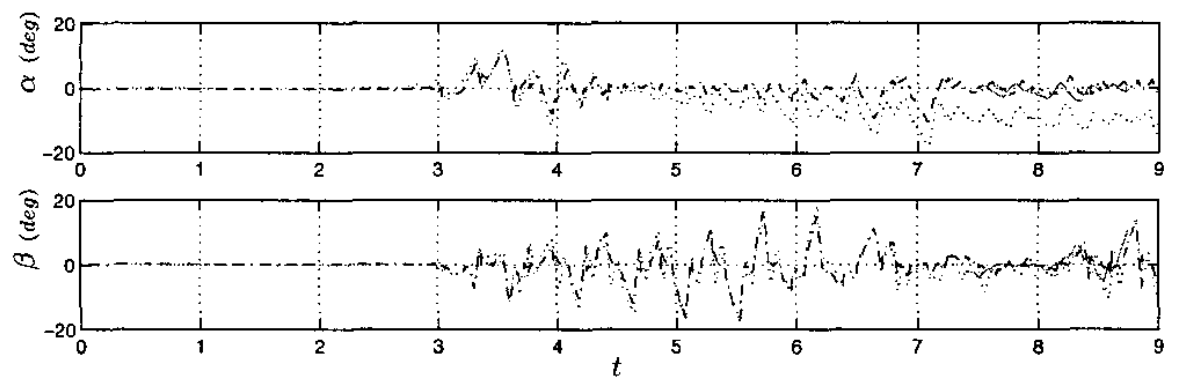

Fig. 3. Pitch $(\alpha)$ and roll $(\beta)$ measured by GTMS (green solid line) and compuled according to our sensor combination scheme (blue dash-dotted line) as compared to direct gyro integrator output (magenta dot line). Note that GTMS data is only available during the fi nal portion of the run when the rohot is within its ficld of vicw.

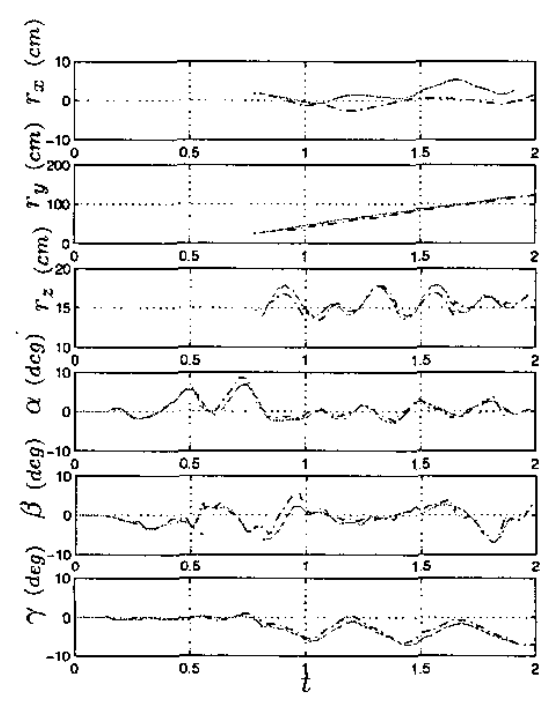

Fig. 4. Body States measured by GTMS (grecn solid line) and computed according to our algorithm (bluc dash-dotted line).

the other end. While the result of orientation is presented over the whole time line, that of COM translation is shown only within stable jogging period without initial transient phase from standstill (around $0.5 \mathrm{sec}$ ) because of the current algorithm's inability to handle unsteady locomotion transients.

Table III summarizes the outcome of 10 runs with mean and standard deviation (std). Small RMS error values (with mean error in angular states less than 2 degrees and in vertical translation less than $1 \mathrm{~cm}$ ) compared to the robot size $(50 \mathrm{~cm} \times 25 \mathrm{~cm} \times 15 \mathrm{~cm})$ indicate successful body state computation, which can also be quantified by $\xi$, the ratio of RMS error of each state to the noise floor of GTMS introduced in Section IV-A, as well as by $\zeta$, the ratio of RMS error of translational state to the robot length $(50 \mathrm{~cm})$. The orientation components exhibit good performance as evidenced by $\xi$-values well under the GTMS noise floor. In contrast, translation state components during non-tripod intervals that are estimated using the purely predictive naive constant-speed and ballistic models exhibit relatively worse performance due to model error, especially in the fore-aft $\left(r_{y}\right)$ and lateral $\left(r_{x}\right)$ directions which depends on
TABLE III

RMS RIGID BODY STATE ESTIMATOR ERRORS IN CONFIGURATION RELATIVE TO GTMS MEASLREMENTS

\begin{tabular}{|c|c|c|c|c|c|c|c|}
\hline Trial & \multicolumn{6}{|c|}{ Statc } & Acrial phase \\
\hline & $\begin{array}{c}r_{x} \\
\text { (cm) }\end{array}$ & $\begin{array}{c}r_{y} \\
(\mathrm{~cm})\end{array}$ & $\begin{array}{l}r_{z} \\
(\mathrm{~cm})\end{array}$ & $\begin{array}{c}\alpha \\
(d c g)\end{array}$ & $\begin{array}{c}\beta \\
\text { (dig) }\end{array}$ & $\begin{array}{c}\gamma \\
\text { (deg) }\end{array}$ & (\%) \\
\hline \multicolumn{8}{|c|}{ Confi guration estimation RMS crror } \\
\hline mean & 2.54 & 4.60 & 0.84 & 0.90 & 1.57 & 1.01 & 26.2 \\
\hline std & 0.60 & 0.61 & 0.12 & 0.16 & 0.29 & 0.15 & 2.6 \\
\hline \multicolumn{8}{|c|}{$\xi$, Ralio of RMS error (mean) shown above 10 GTMS noise foor } \\
\hline & 5.08 & 9.21 & 1.67 & 0.33 & 0.56 & 0.78 & \\
\hline \multicolumn{8}{|c|}{$\zeta$, Ratio $(\%)$ of RMS error (mean) shown above to robot body length $(50 \mathrm{~cm})$} \\
\hline & 5.08 & 9.21 & 1.67 & & & & \\
\hline
\end{tabular}

state history as well as unmodeled activity in the horizontal plane where slippage has significant effect. Translation state components exhibit $\zeta$ values less than $10(\%)$ error compared to robot length suggesting an acceptable performance for the preliminary first trial of this naive sensor combination metohd. In addition, despite having the biggest RMS error, the percentage error of $r_{y}$ is actually less than $6(\%)$ if considering forwarding distance is about $87 \mathrm{~cm}$ in average detailed in Table IV. It's not surprising that the results are worse than that of similar experiments while robot operating in walking gait (i.e. without aerial phase) [23] because of more dynamical behavior in jogging locomotion as well as the implementation of velocity states in algorithm. In walking case the algorithm can be constructed by pure kinematic relations without any velocity states but yields good body pose estimation.

Table IV compares our leg sensor and gyro based odometry ${ }^{9}$ estimates with sensorless schemes by reference to discrepancies with GTMS measurements of elapsed distance ${ }^{10}$. With no sensing apart from motor shaft measurements, "blind odometry" estimates result from counting the number of leg cycles and multiplying by a previously calibrated "distance-per-cycle" constant. Of course, this is the traditional approach to odometry in wheeled vehicles as well. We ran calibration tests for RHex by counting the number of motor shaft cycles over the same long fat surface to get the best possible conversion constant. The table presents discrepancies, $\kappa_{s}(\%)(=|\Delta \nu| / \nu)$, as a percentage of the GTMS measured elapsed distance, $\nu$, for

9For purpose of this paper. "odometry" denotes the COM translation in fore/aft dircetion, $r_{y}$ between initiate point to the end of run.

${ }^{10}$ We thank Dr. Johann Borenstein for suggesting this comparison to 
TABLE IV

ODOMETRY ERROR RELATIVE TO GTMS MEASUREMENTS

\begin{tabular}{|c|c|c|c|c|c|c|}
\hline Trial no. & \multicolumn{9}{|c|}{ Mcasures } \\
\hline & \multicolumn{2}{|c|}{ GTMS reference } & \multicolumn{2}{|c|}{ Sensor-based } & \multicolumn{2}{|c|}{ Sensorless } \\
\hline & $\begin{array}{c}\nu \\
(\mathrm{cm})\end{array}$ & $\begin{array}{c}\dot{\nu} \\
(\mathrm{cm} / \mathrm{s})\end{array}$ & $\begin{array}{c}\left|\Delta \nu_{s}\right| \\
(\mathrm{cm})\end{array}$ & $\begin{array}{c}\kappa_{s} \\
(\%)\end{array}$ & $\begin{array}{c}\mid \Delta \nu_{l} \\
(\mathrm{~cm})\end{array}$ & $\begin{array}{c}\kappa_{l} \\
(\%)\end{array}$ \\
\hline mean & 86.8 & 81.2 & 1.7 & 2.0 & 5.6 & 6.6 \\
sld & 6.6 & 1.5 & 1.2 & 1.5 & 2.0 & 2.5 \\
\hline
\end{tabular}

both odometry methods: sensorless and sensor-based. The results show that in each data the leg sensor and gyro based odometry is better, by a factor of 3 , on average, than the blind predictions of the open loop scheme.

\section{CONCLUSION}

We have introduced a continuous time full body state estimator for a hexapod robot executing a jogging gait (i.e. with aerial phase) on level ground based on a naive sensor combination algorithm using inputs from a leg pose sensor and gyro. We have implemented the algorithm on RHex and evaluated the performance with respect to an independent visual ground truth measurement system (GTMS). For orientation state components, small RMS error less than 2 (deg) well within the GTMS noise floor indicates good performance. For translation state components, performance, while acceptable, is not as impressive (most likely due to the absence of the accelerometer component of the IMU), exhibiting less than $10(\%)$ error compared to body length. In a "sample" application - odometry along a run this combined sensor system outperforms by a factor of three the alternative sensorless "average distance per stride" estimate.

Combining the leg pose sensor and gyro data significantly ameliorates the accumulating integrator drift associated with a gyro alone. On the other hand, without gyro's complementary data supplements, the leg pose sensor alone isn't able to construct continuous time full body state estimation because of the requirement of orientation state in non-tripod intervals and yaw state in tripod stance interval to recalibrate the tripod coordinate system back to world coordinates. Under current circumstance the full body state can only be achieved by combining both sensor data as the minimum requirement.

The present early version of this sensor combination algorithm seems not yet to deserve the "sensor fusion" designation because it makes only heuristic use in an intuitive manner of prior knowledge regarding which sensors perform capably under what circumstances. In any case, our present hardware suite, still missing the accelerometer bank traditionally associated with an IMU, doesn't seem to yet have enough sensor inputs to support a "full sensor fusion" algorithm that would combine two sets of independently working sensors using statistics about their respective accuracies to drive an appropriate stochastic dynamical model. Work in progress on RHex is addressing the need for a reasonably high performance accelerometer bank, and we hope to report on the performance improvements that result from an expanded sensory suite along with a statistically informed estimator that uses it.

\section{ACKNOWLEDGMENT}

We thank Sarjoun Skaff and Al Rizzi for several useful discussions as well as the access to their rate gyro integrator implementation on RHex. We thank Gregory
Sharp for his Ground Truth Measurement System implementation. We also thank Joel D. Weingarten and Joseph Raisanen for jogging gait tuning. This work is supported by DARPA/SPAWAR Contract N66001-00-C-8026 and N66001-03-C-8045.

\section{REFERENCES}

[1] M. Buchler, U. Saranli, and D. E. Koditschek. Single actuator per eg robotic hexapod. United States Patent 6,481,513, 2002. McGil University, The Regents of the University of Michigan. USA

[2] U. Saranli, M. Buehler, and D. E. Koditschek. Rhex - a simple and highly mobile hexapod robot. International Joumal of Robotics Reseanch, 20(7):616-631, 2001.

[3] J. D. Weingarten, R. E. Groff, and D. E. Koditschek. Automated gait generation and optimization for legged robots. In Proc. IEEE Int. Conf. Robotics and Automation, volume 3, pages 2153-2158, 2004.

[4] E. Z. Moore and M. Buchler. Stable stair climbing in a simple hexapod. In 4 th International Conference on Climbing and Walking Robos, pages 603-610, 2001.

[5] E. Z. Moore, D. Campbell, F. Grimminger, and M. Buebler. Reliable stair climbing in the simple hexapod 'rhex'. In Proc. IEEE Int. Conf Stair climbing in the simple hexapod 'rhex'. In Proc. IEEE Int.

[6] D. Campbell and M. Buehler. Stair descent in the simple hexapod 'rhex'. In Proc. IEEE Int. Conf. Robotics and Automation, volume 1, pages $1380-1385,2003$.

[7] H. Komsuoglu, D. McMordic, U. Saranli, N. Moore, M. Buchler and D. E. Koditschek. Proprioception based behavioral advances in a hexapod robot. In Proc. IEEE Int. Conf. Robotics and Automation. volume 4, pages 3650-3655, Seoul, Korea, 2001.

[8] U. Saranli and D. E. Koditschek. Back fips with a hexapodal robot. In Proc. IEEE Int. Conf. Robotics and Automation, volume 3, pages In Proc. IEEE Int.

[9] U. Saranli. Dynamic Locomotion in a Hexapod Robot. PhD thesis. Univerisity of Michigan, August 2002.

[10] P.-C. Lin, H. Komsuoglu, and D. E. Koditschek. A leg confi guration sensory system for dynamical body state estimates in a hexapod robot. In Proc. IEEE Int. Conf. Robotics and Automation, volume 1 , pages $1391-1396,2003$

[11] R. Altendorfer, N. Moore, H. Komsuoglu, H. B. Brown, D. McMordie, U. Saranli. R. Full, and D. E. Koditschek. Rhex: A biologically inspired hexapod runncr. Altonomous Robots, 11(3):207-213, 2001 .

[12] P.-C. Lin, H. Komsuoglu, and D. E. Koditschek. A leg confi guration measurement system for full body pose estimates in a hexapod robot. IEEE Trans. Robor., 2004. submitted for review.

[13] P. J. Escamilla-Ambrosio and N. Mort. A hybrid kalman fi lter furzy logic architecture for multisensor data fusion. In Int. Symp. Intelligent Control, pages 364-369, 2001.

[14] M. Abdelrahman and $P$, Abdelrahman. Integration of multiple sensor fusion in controlled design. In American Control Conf.. pages 26092614, 2002.

[15] S. I. Roumeliotis, A. E. Johnson, and J. F. Montgomery. Augmenting inertial navigation with imagc-based motion estimation. In Proc. IEEE Int. Conf. Robotics and Automation, 2002.

[16] J. Lobo and J. Dias. Integration of incrtial information with vision. In Proc. Annual Conf. IEEE (IECON), volume 3, pages 1263-1267. 1998.

[17] M. Kam, X. Zhu, and P. Kalata. Sensor fusion for mobile robol navigation. In Proc. IEEE, pages 108-119, 1997.

[18] K. Park, H. Chung, J. Choi, and J. G. Lec. Dead reckoning navigation for an autonomous mobile robot using a differential encoder and a gyroscope. In Int. Conf. Advanced Robotics, pages $441-446,1997$

[19] O. Wijk and H. I. Christensen. Triangulation-based fusion of sonar data with application in robot pose tracking. IEEE Trans. Robot. Automat., 16(6):740-752, 2000.

[20] Sarjoun Skaff, George A Kantor, David Maiwand, and Alfred Rizzi. Inertial navigation and visual line following for a dynamical hexapod robot. In IEEE/RSJ Int Conf. Intelligent Robots and Systems, volume 2, pages 1808-1813, October 2003.

[21] S. H. Phang, R. C. Ren, and K. L. Su. The development of intelligent control system for animal robot using multisensor fusion. In $I E E E$ Int. Conf.Multisensor Fusion and Integration for Intelligent Systems, pages $1-6,2001$

[22] R. C. Ren, S. H. Phang, and K. L. Su. Multilevel multisensor based decision fusion for intelligent animal robot. In Proc. IEEE Int. Conf. Robotics and Automation, volume 4, 2001.

[23] P.-C. Lin, H. Komsuoglu, and D. E. Koditschek. Legged odometry from body pose in a hexapod robot. In Int. Symp. Experimental Robotics, 2004.

[24] M. T. Mason. Mechanics of Robotic Manipitation. The MIT Press, 2001. p. 41-58. 